Pacific Journal of Mathematics

SEMIGROUPS WHOSE LATTICE OF CONGRUENCES IS 


\title{
SEMIGROUPS WHOSE LATTICE OF CONGRUENCES IS BOOLEAN
}

\author{
Howard Hamilton and Thomas Nordahl
}

The commutative semigroups whose lattice of congruences forms a Boolean lattice are determined. They are (i) the null semigroups of order two or less, (ii) the discrete trees, (iii) the groups which are a direct sum of prime order cyclic groups in which no two factors have the same order, (iv) the semigroups which are a one element inflation of a discrete tree, ( $v$ ) the semigroups which are a free product of a discrete tree with zero and a semigroup of type (iii) amalgamated over the trivial semigroup, and (vi) the semigroups which are a one element inflation of a semigroup of type (v).

1. Introduction. In this paper a semilattice will be considered to be an upper semilattice (i.e., for $x, y \in S$ we have $x \leqq y$ if and only if $x y=y$ ). We say that semilattice $S$ is a (discrete) tree if for all $x, y \in S$ with $x \leqq y$ the interval $[x, y]=\{z \in S: x \leqq z \leqq y\}$ is a (finite) chain. For any semigroup $S$ we will let $L(S)$ denote the lattice of congruences of $S$. If $T$ is a subsemigroup of semigroup $S$ and there exists a function $f: S \rightarrow T$ satisfying: (i) the restriction of $f$ to $T$ is the identity mapping and (ii) for $x, y \in S$ we have $x y=f(x) f(y)$, then $S$ is said to be an inflation of $T$. We shall say that $S$ is a one element inflation of $T$ if $S$ is an inflation of $T$ and $[S \backslash T]=1$. Terms which are not defined may be found in [3], [9], [12], [13] or in [1].

We now present some results which are needed for our proofs.

THEOREM 1. (Hamilton [7]) Let $S$ be a semilattice. $L(S)$ is a Boolean lattice if and only if $S$ is a discrete tree.

Let $S$ be a semigroup and let $S=\bigcup_{\alpha \in \Gamma} S_{\alpha}$ be the greatest semilattice decomposition of $S$. If $\alpha<\beta$ in $\Gamma$ and for all $a \in S_{\alpha}$ and $b \epsilon$ $S_{\beta}$ we have $a b=b a=b$ then we say that $S_{\alpha}$ and $S_{\beta}$ are 1-composed. If $S_{\alpha}$ and $S_{\beta}$ are 1-composed for all $\alpha, \beta \in \Gamma$ with $\alpha<\beta$ then we say that $S$ is 1-composed.

THEOREM 2. (Hamilton [8]) If $S$ is a commutative seperative semigroup with $L(S)$ a modular lattice then $S$ is 1-composed.

If a semigroup $S$ is isomorphic to a subdirect product of semigroups $T$ and $U$ we shall write: $S \simeq T \times_{S} U$. If a semigroup $S$ is 
isomorphic (equal) to a subdirect product of a collection, $\left\{A_{i}\right\}_{i \in I}$, we shall write $S \simeq \Pi_{i \in I}^{S} A_{i}\left(S=\Pi_{i \in I}^{S} A_{i}\right)$.

THEOREM 3. (Tanaka [15], [2]) Let $A$ be an algebra such that $L(A)$ is a distributive lattice. Then $L(A)$ is a Boolean lattice if and only if $A$ is isomorphic to some $A^{*}$ where:

(i ) $A^{*}=\prod_{i \in I}^{S} A_{i}$, where the $A_{i}$ are congruence simple algebras and

(ii) any two elements of $A^{*}$ are equal on all but finitely many factors $A_{i}$.

We wish to emphasize the difference between simple and congruence simple. A semigroup is said to be simple if it has no proper ideals. A semigroup is said to be congruence simple if $\iota$, the diagonal relation and $\omega$, the universal relation, are the only congruences on $S$.

We shall write $G \simeq \sum_{i \in I} G_{i}$ to indicate that a group $G$ is the direct sum of groups $\left\{G_{i}\right\}_{i \in I}$.

THEOREM 4. (Burris [2]) Let $G$ be a group. $L(G)$ is a Boolean lattice if and only if $G \simeq \sum_{i \in I} G_{i}$, where the $G_{i}$ are simple groups and the factor $Z_{p}$ does not occur twice for any prime $p$.

Corollary 5. Let $G$ be an Abelian group. $L(G)$ is a Boolean lattice if and only if $G \simeq \sum_{p \in P} Z_{p}$, where $P$ is a set of primes.

Proposition 6. (Birkhoff [1]) The partition lattice on a set $S$ is distributive if and only if $|S| \leqq 2$.

Corollary 7. Let $N$ be a null semigroup. $L(N)$ is distributive if and only if $N$ is a null semigroup of order less than or equal to two if and only if $L(N)$ is a Boolean lattice.

THEOREM 8. (Fountain and Lockley [5]) Let $S$ be a semigroup and $\sigma$ a congruence on $S$. If $L(S)$ is a Boolean (modular, distributive) lattice, then $L(S / \sigma)$ is a Boolean (modular, distributive) lattice.

2. Preliminary results. We shall call a semigroup $S$ a Boolean semigroup if $L(S)$ forms a Boolean lattice.

Lemma 9. Let $S$ be a Boolean semigroup. Every cancellative congruence $\sigma$ on $S$ is a cancellative simple congruence, that is, $S / \sigma$ is a cancellative simple semigroup. 
Proof. Let $\sigma$ be a cancellative congruence on $S$. Suppose that $S / \sigma$ has a proper ideal $I$. Suppose that $|I|=1$ then $S / \sigma$ has a zero and so as $S / \sigma$ is cancellative we have that $S / \sigma$ is trivial and so $\sigma$ would be the group congruence $\omega$. If $|I| \neq 1$ then $\rho_{I}$, the Rees congruence with respect to the ideal $I$, has complement $\tau \in L(S / \sigma)$ as $L(S / \sigma)$ will be a Boolean lattice by Theorem 8. As $\tau \cap \rho_{I}=\iota$ on $S / \sigma$ we see that the restriction of $\tau$ to $I,\left.\tau\right|_{I}$, is the diagonal relation

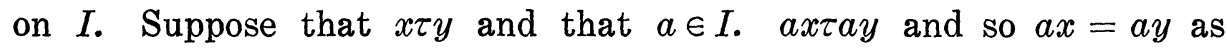
$a x, a y \in I$. As $S / \sigma$ is a cancellative semigroup it follows that $x=y$ and so $\tau$ is the diagonal relation on $S / \sigma$. $\tau$ clearly is not the complement of $\rho_{I}$. Hence $S / \sigma$ has no proper ideals and $\sigma$ is a cancellative simple congruence on $S$.

It is easy to verify that a cancellative simple semigroup with nonempty center is a group (see [11]). Combining this remark with Lemma 9 we obtain:

LEMMA 10. Let $S$ be a Boolean semigroup with nonempty center then every cancellative congruence on $S$ is a group congruence on S. In particular for every commutative semigroup, cancellative congruences are group congruences.

LeMma 11. Let $S$ be a Boolean semigroup with nonempty center. $S$ has a smallest group congruence. In particular, every commutative Boolean semigroup has a smallest group congruence.

Proof. This follows from Lemma 10, as every semigroup has a smallest cancellative congruence.

REMARK 12. The commutative semigroups which are congruence simple are exactly those which are isomorphic to $Z_{p}$ for some prime $p$, a null semigroup of order two, a semilattice of order two or a trivial semigroup.

Proposition 13. Let $S$ be a commutative semigroup. $L(S)$ is a Boolean lattice if and only if $L(S)$ is distributive and $S \simeq$ $T \times_{S} N \times_{S} G$ where $T$ is a discrete tree, $N$ is a null semigroup of order less than or equal to two and $G \simeq \sum_{p * P} Z_{p}$ for some set of primes $P$.

Proof. Let $S$ be a commutative Boolean semigroup. It follows from Remark 12 and Theorem 3 that $S \simeq\left(\Pi_{\sigma \in \Sigma}^{S} S / \sigma\right) \times_{S}\left(\Pi_{\lambda \in A}^{S} S / \lambda\right) \times_{S}$ $\left(\Pi_{\gamma \in \Gamma}^{S} S / \gamma\right)$ where $\Sigma$ is the set of semilattice congruences on $S, \Lambda$ is the set of null congruences on $S$, and $\Gamma$ is the set of group congruences on $S$. Note: we are actually including more than "con- 
gruence simple" congruences in this subdirect decomposition. The intersection of semilattice congruences is a semilattice congruence. The intersection of null congruences is a null congruence. By Lemma 11, the intersection of group congruences is a group congruence. Thus $S \simeq T \times_{S} N \times_{S} G$ where $T$ is a semilattice, $N$ is a null semigroup and $G$ is an Abelian group. By Theorem 8, $L(T)$, $L(N)$, and $L(G)$ are all Boolean lattices. It follows from Corollary 7 that $|N| \leqq 2$. It follows from Theorem 1 that $T$ is a discrete tree. It follows from Corollary 5 that $G \simeq \sum_{p \in P} Z_{p}$ for some set of primes $P$.

Conversely, let us assume that $S \simeq T \times_{S} N \times_{S} G$ where $T$ is a discrete tree, $N$ is a null semigroup with $|N| \leqq 2$ and $G \simeq \sum_{p \in P} Z_{p}$ for some set of primes $P$ and $L(S)$ is a distributive lattice. In order to apply Theorem 3 we need to show that $T, N$, and $G$ satisfy the conditions (i) and (ii) of Theorem 3. As $T$ is a discrete tree it follows from Theorem 1 that $L(T)$ is a Boolean lattice. By Theorem 3 we have that $T$ satisfies conditions (i) and (ii) of Theorem 3. As $N$ is a null semigroup of order less than or equal to two, $N$ clearly satisfies conditions (i) and (ii) of Theorem 3. $G \simeq \sum_{p \in P} Z_{p}$ also clearly satisfies the conditions (i) and (ii) of Theorem 3 . It follows that $S$ satisfies the conditions (i) and (ii) of Theorem 3 and so by Theorem 3 we have that $L(S)$ is a Boolean lattice.

Let $E$ be a semigroup and let $\left\{A_{i}: i \in I\right\}$ be a collection of semigroups and suppose that for each $i \in I$ there exists $\lambda_{i}$ a monomorphism of $E$ into $A_{i}$. A free product of $\left\{A_{i}: i \in I\right\}$ with amalgamated subsemigroup $E$ is a semigroup $B$ possessing a system of monomorphisms $\left\langle\sigma_{i}\right\rangle i \in I$ where $\sigma_{i}$ maps $A_{i}$ into $B$ satisfying:

(i) $\sigma_{i}^{0} \lambda_{i}=\sigma_{j}^{0} \lambda_{j}$ for all $i, j \in I$,

(ii) $\left[\bigcup_{i \in I} \sigma_{i}\left(A_{i}\right)\right]=B$ (where $[X]$ denotes the semigroup generated by $X$, and

(iii). if $C$ is a semigroup and for each $i \in I, \phi_{i} \in \operatorname{HOM}\left(A_{i}, C\right)$ is given so that $\phi_{i}^{0} \lambda_{i}=\phi_{j}^{0} \lambda_{j}$ for all $i, j \in I$, then there exists $\phi \in \operatorname{HOM}(B$, $C)$ satisfying $\phi_{i}=\phi \circ \sigma_{i}$ for all $i \in I$.

Let $S$ be a semigroup with identity, $e$, and let $T$ be a semigroup with zero, 0 . Let $E=\{1\}$ be the trivial semigroup. Let $\lambda_{1}$ : $E \rightarrow S$ be given by $\lambda_{1}(1)=e$ and let $\lambda_{2}: E \rightarrow T$ be given by $\lambda_{2}(1)=0$. Let $B=S \bigcup_{1} T$ be the set union of $S$ and $T$ except that 0 and $e$ are identified. Define a product in $B$ by:

$$
x \circ_{B} y=\left\{\begin{array}{l}
x \circ_{S} y \text { if } x, y \in S, \text { where } \circ_{S} \text { is the operation in } S \\
x \circ_{T} y \text { if } x, y \in T, \text { where } \circ_{T} \text { is the operation in } T \\
x \text { if } x \in S, y \in T \\
y \text { if } x \in T, y \in S .
\end{array}\right.
$$


We define $\sigma_{1}: S \rightarrow B$ and $\sigma_{2}: T \rightarrow B$ by $\sigma_{1}(s)=s$ and $\sigma_{2}(t)=t$ for all $s \in S, t \in T$. Note that $\sigma_{1}(e)=\sigma_{2}(0)$. It is easy to see that the semigroup $B$ is the free product of $S$ and $T$ amalgamated over $\{1\}$, so as to identify $\dot{e}$ and 0 .

Proposition 14. Let $S$ be a semigroup with identity, $e$, and let $T$ be a semigroup with zero, 0 . Let $B$ be the free product of $S$ and $T$ amalgamated over the trivial semigroup $\{1\}$. Then we have $L(B) \simeq L(S) \times L(T)$.

Proof. Let $\rho \in L(B)$. We define $\phi: L(B) \rightarrow L(S) \times L(T)$ by $\phi(\rho)=$ $\left(\left.\rho\right|_{S},\left.\rho\right|_{T}\right)$. We define the inverse map $\psi: L(S) \times L(T) \rightarrow L(B)$ by $\psi(\sigma, \tau)=\rho$ where:

$$
x \rho y \text { if }\left\{\begin{array}{l}
x, y \in S \text { and } x \sigma y, \\
x, y \in T \text { and } x \tau y, \\
x \in S, y \in T \text { and } x \sigma e, 0 \tau y \text { or } \\
x \in T, y \in S \text { and } x \tau 0, \text { ery (recall } e=0 \text { in } B \text { ). }
\end{array}\right.
$$

Let $\rho \in L(B)$ and suppose $x \rho y$ where $x \in S$ and $y \in T$. We see that $x=x e \rho y e=y 0=0=e$ and so $x \rho e$. Also we see that $0 \rho \times \rho y$ and so $0 \rho y$. Conversely, if $x \in S$ and $y \in T$ with $x \rho e$ and $0 \rho y$, then we have $x \rho y$ by the transitivity of $\rho$. Thus $\rho$ is determined by its restrictions to $S$ and $T$. We see that $\psi$ is the inverse of $\phi$ and so $L(B) \simeq L(S) \times L(T)$.

3. The general case. Let $S$ be a commutative Boolean semigroup. By Proposition 13, we have that $S \simeq T \times_{S} N \times_{S} G$ where $T$ is a discrete tree, $N$ is a null semigroup with $|N| \leqq 2$ and $G \simeq$ ${ }_{p} \sum_{P} Z_{p}$ for some set of primes $P$. In what follows we shall consider $S$ to be a subsemigroup of the direct product $T \times N \times G$. Let $\pi: T \times N \times G \rightarrow T \times G$ be the projection homomorphism. $U=\pi(S)$ is the greatest separative homomorphic image of $S$. $U$ is a subsemigroup of the direct product $T \times G$ which is a subdirect product of $T$ and $G$. For each $\alpha \in T$ we let $U_{\alpha}=\{(\alpha, g) \in[\alpha] \times G:(\alpha, g) \in U\}$. We see that $U=\bigcup_{\alpha \in T} U_{\alpha}$ is a semilattice decomposition of $U$ which is in fact the greatest semilattice decomposition of $U, U$ is a commutative separative Boolean semigroup. It follows from Theorem 2 that $U$ is 1-composed. Suppose $\alpha, \beta \in T$ with $\alpha<\beta$ and that $(\alpha, g),(\beta, h) \in U$. Then as $U$ is 1 -composed we see that: $(\beta, h)=$ $(\alpha, g)(\beta, h)=(\alpha \beta, g h)=(\beta, g h)$. Hence $g h=h$ and as $G$ is a group we have that $g=e$, the identity of $G$. We can conclude that if $G$ is not trivial that $T$ has a zero, 0 , with $U_{0}=\{(0, g) ; g \in G\} \simeq G$ and $U_{\beta}$ is trivial for all other $\beta \in T$. If $G$ is trivial then $U \simeq T$ is a 
discrete tree. If $G$ is not trivial, $U$ is isomorphic to the free product of $G$ and $T$ amalgamated over the trivial semigroup $\{1\}$ so as to identify $0 \in T$ and $e \in G$. By Proposition 15, $L(U) \simeq L(G) \times L(T)$. By Theorem $1, L(T)$ is a Boolean lattice. By Corollary $5, L(G)$ is a Boolean lattice and so $L(U)$ is a Boolean lattice. We have shown:

THEOREM 15. Let $U$ be a commutative separative semigroup. $L(U)$ is Boolean if and only if

(i) $U$ is a discrete tree or

(ii) $U$ is a free product of $G \simeq \sum_{p \in P} Z_{p}$ ( $P$ a nonempty set of primes) and $T$, a discrete tree with zero, amalgamated over the trivial semigroup.

We return to the general case where $S$ is our commutative Boolean semigroup and $S$ is a subsemigroup of $T \times N \times G$. We now consider the projection $h: T \times N \times G \rightarrow T \times N$. A semigroup is called group free if all of its subgroups are trivial. It is easy to see that the intersection of group free congruences is a group free congruence and so every semigroup has a greatest group free homomorphic image. $V=h(S)$ is the greatest group free homomorphic image of $S$. For each $\gamma \in T$ we let $V_{\gamma}=\{(\gamma, x) \in\{\gamma\} \times N:(\gamma, x) \in$ $V\} . \quad V=\bigcup_{\alpha \in T} V_{\alpha}$ is the greatest semilattice decomposition of $V$. Each $V_{\alpha}$ is either trivial or a null semigroup of order 2. Let $\alpha \in T$. We denote the zero of $V_{\alpha},(\alpha, 0)$ by $0_{\alpha}$. If $V_{\alpha}$ is not trivial we denote the other element, $(\alpha, a)$, by $a_{\alpha}$.

LeMma 16. Let $V$ be a group free, commutative Boolean semigroup and let $V=\bigcup_{\alpha \in T} V_{\alpha}$ be the greatest semilattice decomposition of $V$. If $\alpha, \beta \in T$ and $\alpha<\beta$ then $V_{\alpha}$ and $V_{\beta}$ are not both nontrivial.

Proof. We can see that $V$ is an inflation of $T \times\{0\} \simeq T$. For each $\alpha \in T, V_{\alpha}=\left\{0_{\alpha}, a_{\alpha}\right\}$ or $V_{\alpha}=\left\{0_{\alpha}\right\}$. Suppose $\alpha, \beta \in T$ with $\alpha<\beta$ and $V_{\alpha}=\left\{0_{\alpha}, a_{\alpha}\right\}$ and $V_{\beta}=\left\{0_{\beta}, a_{\beta}\right\}$. We shall see that this assumption leads to a contradiction. Let us first show that $V_{\alpha} \cup V_{\beta}$ is a homomorphic image of $V$ and so $L\left(V_{\alpha} \cup V_{\beta}\right)$ is a Boolean lattice by Theorem 8. Let $V^{\alpha}=\bigcup_{r \leqq \alpha} V_{r}$. We define $f: V \rightarrow V_{\alpha} \cup V_{\beta}$ as follows:

(i) if $x \in V^{\alpha} \backslash\left\{a_{\alpha}\right\}$ then $f(x)=0_{\alpha}$,

(ii) $f\left(a_{\alpha}\right)=a_{\alpha}$,

(iii) if $x \in V \backslash\left(V^{\alpha} \cup\left\{a_{\beta}\right\}\right)$ then $f(x)=0_{\beta}$ and

(iv) $f\left(a_{\beta}\right)=a_{\beta}$.

We note that $\left\{0_{\alpha}\right\}$ is an ideal of $V^{\alpha}$ and that $\left\{a_{\alpha}\right\} V^{\alpha}=V^{\alpha}\left\{a_{\alpha}\right\}=\left\{0_{\alpha}\right\}$ and so the restriction of $f$ to $V^{\alpha}$ is a homomorphism. We note 
that $V \backslash V^{\alpha}=I$ is a prime ideal with $\left\{a_{\beta}\right\} V=V\left\{a_{\beta}\right\} \subseteq I$ and so $V \backslash\left(V^{\alpha} \cup\left\{a_{\beta}\right\}\right)$ is an ideal of $V$. The function $f$ is thus a homomorphism and $f(V)=V_{\alpha} \cup V_{\beta}$ is a Boolean semigroup. Let us define the relations $\sigma, \rho_{1}$, and $\rho_{2}$ on $f(V)$ as follows:

$$
\begin{gathered}
x \sigma y \text { if }\left\{\begin{array}{l}
x, y \in\left\{0_{\alpha}, 0_{\beta}\right\} \text { or } \\
x, y \in\left\{a_{\alpha}, a_{\beta}\right\},
\end{array}\right. \\
x \rho_{1} y \text { if }\left\{\begin{array}{l}
x, y \in\left\{0_{\alpha}, a_{\alpha}\right\} \text { or } \\
x=y, \text { and }
\end{array}\right. \\
x \rho_{2} y \text { if }\left\{\begin{array}{l}
x, y \in\left\{0_{\alpha}, a_{\alpha}\right\} \text { or } \\
x, y \in\left\{0_{\beta}, a_{\beta}\right\} .
\end{array}\right.
\end{gathered}
$$

$\sigma, \rho_{1}$, and $\rho_{2}$ are clearly congruences on $f(V)$ and $\sigma \cap \rho_{1}=\sigma \cap \rho_{2}=$ ¿, $\sigma \vee \rho_{1}=\sigma \vee \rho_{2}=\omega$. So $L\left(V_{\alpha} \cup V_{\beta}\right)$ contains $N_{5}$, the nonmodular lattice of order 5 , which is a contradiction to the fact that $L\left(V_{\alpha} \cup\right.$ $\left.V_{\beta}\right)$ is a Boolean lattice. Thus $V_{\alpha}$ and $V_{\beta}$ are not both nontrivial.

Lemma 17. Let $V$ be a commutative, group-free, Boolean semigroup and let $V=\bigcup_{\alpha \in T} V_{\alpha}$ be the greatest semilattice decomposition of $V$. If $\alpha, \beta \in T$ are incomparable elements of $T$ then $V_{\alpha}$ and $V_{\beta}$ are not both nontrivial.

Proof. Suppose by way of contradiction that there exist incomparable elements $\alpha, \beta \in T$ with $V_{\alpha}=\left\{0_{\alpha}, a_{\alpha}\right\}$ and $V_{\beta}=\left\{0_{\beta}, a_{\beta}\right\}$. By Lemma 16, $V_{\alpha \beta}=\left\{0_{\alpha \beta}\right\}$ is trivial. We shall see that $V_{\alpha} \cup V_{\beta} \cup V_{\alpha \beta}$ is a homomorphic image of $V$ and so $L\left(V_{\alpha} \cup V_{\beta} \cup V_{\alpha \beta}\right)$ is a Boolean lattice by Theorem 8. We define $f: V \rightarrow V_{\alpha} \cup V_{\beta} \cup V_{\alpha \beta}$ as follows:

(i) if $x \in V^{\alpha} \backslash\left\{a_{\alpha}\right\}$ then $f(x)=0_{\alpha}$,

(ii) $f\left(a_{\alpha}\right)=a_{\alpha}$,

(iii) if $x \in V^{\beta} \backslash\left\{a_{\beta}\right\}$ then $f(x)=0_{\beta}$,

(iv) $f\left(a_{\beta}\right)=a_{\beta}$, and

(v) if $x \in V \backslash\left(V^{\alpha} \cup V^{\beta}\right)$ then $f(x)=0_{\alpha \beta}$.

It follows that $f$ is a homomorphism as $V \backslash\left(V^{\alpha} \cup V^{\beta}\right)$ is an ideal of $V,\left\{0_{\alpha}\right\}$ is an ideal of $V^{\alpha},\left\{0_{\beta}\right\}$ is an ideal of $V^{\beta}$ and as $V^{\alpha}\left\{a_{\alpha}\right\}=$ $\left\{0_{\alpha}\right\}$ is an ideal of $V^{\alpha},\left\{0_{\beta}\right\}$ is an ideal of $V^{\beta}$ and as $V^{\alpha}\left\{a_{\alpha}\right\}=\left\{0_{\alpha}\right\}$ and $V^{\beta}\left\{a_{\beta}\right\}=\left\{0_{\beta}\right\}$. follows:

We define relations $\rho_{1}, \rho_{2}$, and $\sigma$ on $f(V)=V_{\alpha} \cup V_{\beta} \cup V_{\alpha \beta}$ as $x \sigma y$ if $\left\{\begin{array}{l}x, y \in\left\{a_{\alpha}, a_{\beta}\right\} \text { or } \\ x, y \in\left\{0_{\alpha}, 0_{\beta}, 0_{\alpha \beta}\right\}\end{array}\right.$ 


$$
\begin{aligned}
& x \rho_{1} y \text { if }\left\{\begin{array}{l}
x, y \in\left\{0_{\alpha}, a_{\alpha}\right\} \text { or } \\
x, y \in\left\{0_{\beta}, a_{\beta}\right\} \text { or } \\
x=y
\end{array}\right. \\
& x_{2} y \text { if }\left\{\begin{array}{l}
x, y \in\left\{0_{\beta}, a_{\beta}\right\} \text { or } \\
x=y .
\end{array}\right.
\end{aligned}
$$

It is immediate that these three relations are all congruence relations and that $\sigma \rho_{1}=\sigma \rho_{2}=\iota$ and $\sigma \rho_{1}=\sigma \rho_{2}=\omega$. We see that $N_{5}$, the nonmodular lattice, is a sublattice of $L\left(V_{\alpha} \cup V_{\beta} \cup V_{\alpha \beta}\right)$ contradicting the fact $L\left(V_{\alpha} \cup V_{\beta} \cup V_{\alpha \beta}\right)$ is modular, in fact a Boolean lattice. It follows that $V_{\alpha}$ and $V_{\beta}$ cannot both be nontrivial.

THEOREM 18. $V$ is a group-free, commutative, Boolean semigroup if and only if $V$ is either a discrete tree or is a one element inflation of a discrete tree.

Proof. Let $V$ be a group-free, commutative, Boolean semigroup and let $V=\bigcup_{\alpha \in T} V_{\alpha}$ be the greatest semilattice decomposition of $V$. By Lemmas 16 and 17 it follows that $V_{\alpha}$ is nontrivial for at most one $\alpha \in T$. Hence $V$ is either a discrete tree or a one element inflation of a discrete tree as $V$ is a subsemigroup of $T \times N$.

Conversely, if $V$ is a discrete tree we know that $L(V)$ is a Boolean lattice by Theorem 1 . We thus assume that $V$ is a one element inflation of a discrete tree $T$ (identifying $T$ and $T \times\{0\}$ ). Then $V=\bigcup_{\alpha \in T} V_{\alpha}$ where for some $\alpha \in T, V_{\alpha}=\left\{0_{\alpha}, a_{\alpha}\right\}$ and $V_{\beta}$ is trivial for all $\beta \in T \backslash\{\alpha\}$. We note that for all $x \in V, x a_{\alpha}=x 0_{\alpha}$ by the definition of an inflation. We shall now show that $L(V) \simeq L(T) \times$ $L\left(V_{\alpha}\right)$. Let $\phi: L(V) \rightarrow L(T) \times L\left(V_{\alpha}\right)$ be given by $\phi(\rho)=\left(\left.\rho\right|_{T},\left.\rho\right|_{V_{\alpha}}\right)$. Recall that we are identifying $T$ with $T \times\{0\} . \quad \phi$ is clearly an order preserving map.

Let $\rho \in L(V)$ and suppose $x \rho a_{\alpha}$ for some $x \in V \backslash\left\{a_{\alpha}\right\}$. We find that $a_{\alpha} \rho x=x^{2} \rho a_{\alpha}^{2}=0_{\alpha}$. Thus $x \rho a_{\alpha}$ implies that $x \rho 0_{\alpha}$ and $0_{\alpha} \rho a_{\alpha}$. Conversely, if $x \rho 0_{\alpha}$ and $0_{\alpha} \rho a_{\alpha}$ we have $x \rho a_{\alpha}$. We see that $\rho$ is determined by its restrictions to $T$ and $V_{\alpha}$. We can now define the inverse to $\phi, \psi$. $\psi: L(T) \times L\left(V_{\alpha}\right) \rightarrow L(V)$ by $\psi(\sigma, \tau)=\rho$ where

$$
x \rho y \text { if }\left\{\begin{array}{l}
x, y \in T \text { and } x \sigma y, \text { or } \\
x, y \in V_{\alpha} \text { and } x \tau y, \text { or } \\
x \in T, y=a_{\alpha} \text { and } x \sigma 0_{\alpha}, 0_{\alpha} \tau a_{\alpha}, \text { or } \\
x=a, y \in T \text { and } x \tau 0_{\alpha}, 0_{\alpha} \sigma y .
\end{array}\right.
$$

$\psi$ is clearly a lattice homomorphism which is the inverse of $\phi$ and so $L(V) \simeq L(T) \times L\left(V_{\alpha}\right)$. As $T$ is a discrete tree, $L(T)$ is a Boolean 
lattice by Theorem 1. $L\left(V_{\alpha}\right) \simeq\{\iota, \omega\}$ and so is a Boolean lattice. $L(V)$ is a direct product of Boolean lattices and so is a Boolean lattice.

We return again to our general case: $S$ is a commutative, Boolean semigroup which we can identify as a subsemigroup of some $T \times N \times G$.

TTHEOREM 19. $S$ is a commutative, Boolean semigroup if and only if $S$ is one of the following:

(i ) a null semigroup of order two or less,

(ii) a discrete tree,

(iii) isomorphic to $\sum_{p \in P} Z_{p}$ for some set of primes $P$,

(iv) a one element inflation of a discrete tree,

(v) a free product of a discrete tree with zero and a group isomorphic to $\sum_{p \in P} Z_{p}, P$ a nonempty set of primes, amalgamated over the trivial semigroup so as to identify the identity of the group and the zero of the discrete tree.

(vi) a one element inflation of a semigroup of type $(v)$.

Proof. Let $S$ be a commutative, Boolean semigroup. We assume as before that $S$ is a subsemigroup of $T \times N \times G$. In the case that $N$ is trivial it follows from Theorem 15 that $S$ is of type (ii) or of type (v). In the case that $G$ is trivial it follows from Theorem 18 that $S$ is of type (ii) or of type (iv).

Let us consider the case when $N$ and $G$ are both nontrivial. We know that $G \simeq \sum_{p \in P} Z_{p}$, where $P$ is a nonempty set of primes. By Theorem 15, $T$ is a discrete tree with zero. Let $k: T \times N \times G \rightarrow T$, $\pi: T \times N \times G \rightarrow T \times G$ and $h: T \times N \times G \rightarrow T \times N$ be the projection homomorphisms. For each $\alpha \in T$ we let $S_{\alpha}=k^{-1}(\alpha) \cap S$. $S=\bigcup_{\alpha \in T} S_{\alpha}$ is the greatest semilattice decomposition of $S$. Let $0 \in T$ be the zero of $T$. It follows from Theorem 15 that $\pi\left(S_{0}\right)=\{0\} \times G \simeq G$ and $\pi\left(S_{\beta}\right)$ is trivial for all $\beta \in T \backslash\{0\}$. By Theorem 18, there exists $\alpha \in T$ such that $h\left(S_{\alpha}\right)=\{(\alpha, 0),(\alpha, a)\}$ and $h\left(S_{\beta}\right)$ is trivial for all $\beta \in$ $T \backslash\{\alpha\}$. Thus we see that $S_{\gamma}$ is trivial for all $\gamma \in T \backslash\{\alpha, 0\}$. We consider two cases:

Case (i). We assume $\alpha \neq 0$. In this case it is easy to see that $S$ is a one element inflation of $S \backslash\{(\alpha, a)\}$, which is a semigroup of type (v). $S$ is thus a semigroup of type (vi).

Case (ii). We assume that $\alpha=0$. We have that $S_{0}$ is a subsemigroup of $\{0\} \times N \times G$ and $S_{\beta}$ is trivial for all $\beta \in T \backslash\{0\}$. Let $a$ be the nonzero element and $0_{N}$ be the zero of the null semigroup $N$. We note that if $\beta \neq 0$, that $S_{\beta}=\{(\beta, 0, e)\}$. The set $I=T \times$ $\left\{0_{N}\right\} \times\{e\} \cup\left(\{0\} \times\left\{0_{N}\right\} \times G\right)$ is a proper ideal of $S$. Let $\rho_{I}$ be the Rees congruence on $S$ with respect to the ideal $I . S / \rho_{I} \simeq(\{0\} \times$ 
$\{a\} \times G) \cap S \cup\{0\}$ is a null semigroup of order two as $S / \rho_{I}$ is a Boolean null semigroup and so $|(\{0\} \times\{a\} \times G) \cap S|=1$. Thus $S_{0}=$ $(\{0\} \times N \times G) \cap S$ is a one element null extension of $\{0\} \times\left\{0_{N}\right\} \times G$. Utilizing the fact: $S \subset T \times N \times G$ we see that $S$ is a one element inflation of $S \backslash\{x\}$ where $\{x\}=(\{0\} \times\{a\} \times G) \cap S . \quad S \backslash\{x\}$ is a semigroup of type (v) and so $S$ is a semigroup of type (vi).

Conversely, let us assume that $S$ is a semigroup of type (i), (ii), (iii), (iv), (v) or (vi). We shall show that $L(S)$ is a Boolean lattice. By Theorem 18, semigroups of type (i), (ii), and (iv) are Boolean. By Theorem 15, semigroups of type (iii) and (v) are Boolean. We can thus assume that $S$ is a semigroup of type (vi). There thus exists $x \in S$ such that $S \backslash\{x\}$ is isomorphic to the free product of a discrete tree $T$ with zero, 0 , and a group $G$, isomorphic to $\sum_{p \in P} Z_{p}$ for some nonempty set of primes $P$, amalgamated so as to identify the identity of $G, e$, and the zero of $T$. We shall identify $S \backslash\{x\}$ with $G \cup T$ where $0 \in T$ is identified with $e \in G$ and if $\alpha \in T, g \in G$ we have $\alpha g=g \alpha=g . \quad S=\bigcup_{\beta \in T} S_{\beta}$ where $G \subset S_{0}, x \in S_{\alpha}$ for some $\alpha \in T$ and $S_{\gamma}=\{\gamma\}$ if $\gamma \notin\{\alpha, 0\}$. We consider two cases:

Case (i). We assume that $\alpha \neq 0$ and so $S_{\alpha}=\{x, \alpha\}$ and $S_{0}=$ G. We shall see that $L(S) \simeq L(T) \times L\left(S_{0}\right) \times L\left(S_{\alpha}\right)$. We define a map $\phi: L(S) \rightarrow L(T) \times L\left(S_{0}\right) \times L\left(S_{\alpha}\right)$ by $\phi(\rho)=\left(\left.\rho\right|_{T},\left.\rho\right|_{S_{0}},\left.\rho\right|_{S_{\alpha}}\right)$. Let $\rho \in L(S)$. As $\left|S_{\alpha}\right|=2,\left.\rho\right|_{S_{\alpha}}=\iota$ or $\left.\rho\right|_{S_{\alpha}}=\omega$. Suppose that $x \rho b$ for some $b \in S \backslash S_{\alpha}$. If $b \in T$, then $\alpha=x^{2} \rho b^{2}=b \rho x$. If $b \in G$, then as $G$ is periodic, there exists $n$ such that $b^{n}=b$ and so $x \rho b=b^{n} \rho x^{n}=\alpha$. Hence if $\left.\rho\right|_{S_{\alpha}}=\ell$, we have that the $\rho$ class of $x,[x]_{\rho}=\{x\}$ and if $\left.\rho\right|_{S_{\alpha}}=\omega$, then $[x]_{\rho}=[\alpha]_{\rho}$. Suppose that $u \in S_{0}, y \in S \backslash S_{0}$ and that $u \rho y$. As $u \in S_{0}$ there exists $m$ such that $u^{m}=e$, as $S_{0}$ is the periodic group $G$. If $y \in T$, then $y$ is idempotent and so $e=u^{m} \rho y^{m}=y \rho u$ and so $u \rho e$ and $e=0 \rho y$. If $y=x$, then we have that $[x]_{\rho} \neq\{x\}$ and so $[x]_{\rho}=[\alpha]_{\rho}$, implying that $x \rho \alpha$ and so by transitivity of $\rho, u \rho \alpha$. As $\alpha$ is an idempotent we have by the previous argument that $u \rho e$ and $0=e \rho \alpha$ and so noting that $x \rho \alpha$ we have that $u \rho e$ and $0=$ $e \rho x$. We see that $\rho$ is the transitive closure of $\left.\left.\left.\rho\right|_{T} \cup \rho\right|_{S_{0}} \cup \rho\right|_{S_{\alpha}}$. We define $\psi: L(T) \times L\left(S_{0}\right) \times L(S) \rightarrow L(S)$ by $\psi(\sigma, \tau, \theta)=\rho$ where $\rho$ is the transitive closure of $\left(\sigma \cup \iota_{S}\right) \cup\left(\tau \cup \iota_{S}\right) \cup\left(\theta \cup \iota_{S}\right)$. The product on $S$ insures that $\rho$ will be a compatible relation and so a congruence relation. It is clear that $\phi$ and $\psi$ are order preserving maps which are inverse and so $L(S) \simeq L(T) \times L\left(S_{0}\right) \times L\left(S_{\alpha}\right)$. $\quad T$ is a discrete tree and so by Theorem $1, L(T)$ is a Boolean lattice. $S_{0} \simeq \sum_{p \in P} Z_{p}$ and so by Corollary $5, L\left(S_{0}\right)$ is a Boolean lattice. As $S_{\alpha}$ is a null semigroup of order two, $L\left(S_{\alpha}\right)$ is a Boolean lattice. $L(S)$ is isomorphic to the direct product of Boolean lattices and so is a Boolean lattice itself.

Case (ii). We assume that $\alpha=0$ and so $S_{0}=G \cup\{x\}$ and $S_{\beta}=$ 
$\{\beta\}$ if $\beta \neq 0$. We have shown in the proof of Theorem 15 that $L(S \backslash\{x\}) \simeq L\left(S_{0} \mid\{x\}\right) \times L(T)$ is a Boolean lattice. Let $e$ be the identity of $G$ and let $\rho \in L(S)$. If $[x]_{\rho} \neq\{x\}$, then $x \rho y$ for some $y \neq x$. If $y \in S_{0}$, then $x \rho y=y e \rho x e$ and so $[x]_{\rho}=[x e]_{\rho}$. Suppose that $y \in T$. As $x^{2}$ is an element of periodic group $G$, there exists $n$ such that $x^{n}=e$ and so we have $y=y^{n} \rho x^{n}=e$. We now see that $x e \rho y y=$ $y \rho x$ and so $[x]_{\rho}=[x e]_{\rho}$. Thus given a congruence relation on $S \backslash\{x\}$, there are exactly two ways to extend to a congruence relation on $S$. We can add $\{x\}$ as a singleton class which poses no compatibility difficulties as $x$ behaves multiplicatively as though it were $x e$, an element of $G$, or we could adjoin $x$ to the class containing $x e$ and this poses no compatibility difficulties for the same reason. Thus $L(S) \simeq L(S \backslash\{x\}) \times 2 \simeq L(G) \times L(T) \times 2$, where 2 is the two element Boolean lattice. $L(S)$ is isomorphic to a direct product of Boolean lattices and so is itself a Boolean lattice.

CoRollary 20. $S$ is a commutative Boolean semigroup if and only if $S \simeq T \times_{S} N \times_{S} G$, where $T$ is a discrete tree, $N$ is a null semigroup of order two or less, $G \simeq \sum_{p \in P} Z_{p}$ for some set of primes $P, L(S)$ is a modular lattice and every null homomorphic image of $S$ is of order two or less.

Proof. If one examines the proofs in $\S 3$, he will see that the relevant half of Theorem 15 is based upon the semigroup being 1-composed which followed from modularity. The relevant half of Theorem 18 rests upon Lemmas 16 and 17 whose proofs required only modularity. The relevant half of Theorem 19 essentially rests upon Theorem 15 and Theorem 18 except where we noted that a certain null homomorphic image had order two. We thus have that these conditions are sufficient. The necessity of these conditions follows from Proposition 13.

\section{ADDENDUM}

Lemma 21. Let $T$ be a discrete tree. Then $L(T)$ is isomorphic to $P(A)$, the set of all subsets of the set of atoms of $L(T)$.

Proof. It is known (see p. 171 of [9]) that a Boolean lattice $B$ is isomorphic to $P(A)$ if and only if $B$ is atomic and complete. The lattice of congruences of any algebra is complete. Let $x, y \in T$ such that $x$ covers $y$ (i.e., $x>y$ and there is no $z$ satisfying $x>z>y$ ). We define the congruence $\sigma_{x, y}$ by: 


$$
a \sigma_{x, y} b \text { if }\left\{\begin{array}{l}
a, b \in\{x, y\} \text { or } \\
x=y
\end{array} \text {. The set of atoms of } L(T)\right. \text { is the set }
$$

$\left\{\sigma_{x, y}: x\right.$ covers $\left.y ; x, y \in T\right\}$. It is clear that every congruence contains an atom and so $L(T)$ is atomic. $L(T)$ is thus isomorphic to $P(A)$.

Lemma 22. $P(A) \times P(B) \simeq P(A \cup B)$, for any two disjoint sets $A$ and $B$.

Proof. The maps are given by $(C, D) \rightarrow C \cup D$ and $E \rightarrow(E \cap$ $A, E \cap B)$.

LEMMA 23. If $S \simeq \sum_{p \in P} Z_{p}$ for some nonempty set of primes $P$, then $L(S) \simeq P(P)$.

Proof. The atoms of $L(S)$ are the congruences induced by $Z_{p}$ for some $p \in P$.

Lemma 24. Let $S$ be a one element inflation of a discrete tree T. Then $L(S)$ is isomorphic to $P(A)$ for some set $A$.

Proof. We have shown that $L(S) \simeq L(T) \times 2$ in the proof of Theorem 18. Hence by Lemmas 21 and 22 we see that $L(S)$ is isomorphic to $P(A)$ for some set $A$.

Lemma 25. Let $S$ be a type (v) semigroup of Theorem 19. Then $L(S)$ is isomorphic to $P(A)$ for some set $A$.

Proof. We have shown in the proof of Theorem 15 that $L(S) \simeq$ $L(G) \times L(T)$, where $G$ is a Boolean group and $T$ is a discrete tree with zero. It follows now by Lemmas 21,22 , and 24 that $L(S)$ is isomorphic to $P(A)$ for some set $A$.

Lemma 26. Let $S$ be a type (vi) semigroup of Theorem 19. Then $L(S)$ is isomorphic to $P(A)$ for some set $A$.

Proof. It is shown in the proof of Theorem 19 that $L(S) \simeq$ $L(T) \times L(G) \times 2$, where $T$ is a discrete tree with zero, $G$ is a Boolean group and 2 is the two element Boolean lattice. It follows now by Lemmas 21,22 , and 23 that $L(S)$ is isomorphic to $P(A)$ for some set $A$.

THEOREM 27. Let $S$ be a commutative semigroup. Then $L(S)$ 
is a Boolean lattice if and only if $L(S) \simeq P(A)$ for some set $A$.

Proof. This follows from Theorem 19 and Lemmas 21, 23, 24, 25 , and 26.

The authors would like to express their deep appreciation to the referee for his many helpful suggestions. The authors would also like to thank Professor Boris Schein for pointing out that Zitomirski [16] has characterized inverse semigroups whose lattice of congruences is Boolean.

\section{REFERENCES}

1. G. Birkhoff, Lattice Theory, Amer. Math. Soc. Colloquium publications, Vol. XXV, Providence, Rhode Island, 1948.

2. S. Burris, Separating sets in modular lattices with applications to congruence lattices, to appear.

3. A.H. Clifford and G.B. Preston, Algebraic Theory of Semigroups, Amer. Math. Soc. Surveys No. 7, Providence, Rhode Island, 1961.

4. R.A. Dean and R.H. Oehmke, Idempotent semigroups with distributive right congruence lattices, Pacific J. Math., 14 (1964), 1187-1209.

5. J.B. Fountain and P. Lockley, Semilattices of groups with distributive congruence lattice, Semigroup Forum, 14 (1977),81-91.

6. G. Gratzer, Universal Algebra, van Nostrand, Princeton, New Jersey, 1968.

7. H. Hamilton, Semilattices whose structure lattice is distributive, Semigroup Forum, 8 (1974), 245-253.

8. H. Hamilton, Commutative separative semigroups whose lattice of congruences is modular, to appear.

9. E. Mendelson, Boolean Algebra and Switching Circuits, McGraw Hill Publishing Co., New York, 1970.

10. J. von Neumann, Continuous Geometry, Princeton Univ. Press, 1960.

11. T.E. Nordahl, Cancellative semigroups with nonempty center, Semigroup Forum, 11 (1975), 170-177.

12. R.S. Pierce, Introduction to the Theory of Abstract Algebras, Holt, Rinehart, and Winston, New York, 1968.

13. M. Petrich, Introduction to Semigroups, Merril Publishing Co., Columbus, Ohio, 1972.

14. T. Tamura, The theory of operators on binary relations, Trans. Amer. Math. Soc., 120 (1965), 343-358.

15. T. Tanaka, Canonical subdirect factorization of lattices, J. Sci. Hiroshima Univ., Ser. A-1 16 (1952), 239-246.

16. G.I. Zitomirskii, Generalized groups with the Boolean lattice of congruence relations (Russian), Uporjadoc Mnozestra i Resetki 2 (1974), 18-27.

Received April 26, 1977 and in revised form November 16, 1977.

University of South Carolina

Columbia, SC 29208 



\section{PACIFIC JOURNAL OF MATHEMATICS}

\section{EDITORS}

RICHARD ARENS (Managing Editor)

University of California

Los Angeles, California 90024

C. W. Curtis

University of Oregon

Eugene, OR 97403

C. C. MOORE

University of California

Berkeley, CA 94720

\section{J. DUGUNDJI}

Department of Mathematics University of Southern California Los Angeles, California 90007

R. Finn AND J. Milgram Stanford University Stanford, California 94305

\section{ASSOCIATE EDITORS}

E. F. BeCK ENBACH

B. H. NeUMaNN

F. WOLF

K. Yoshida

\section{SUPPORTING INSTITUTIONS}

UNIVERSITY OF BRITISH COLUMBIA CALIFORNIA INSTITUTE OF TECHNOLOGY UNIVERSITY OF CALIFORNIA MONTANA STATE UNIVERSITY UNIVERSITY OF NEVADA, RENO NEW MEXICO STATE UNIVERSITY OREGON STATE UNIVERSITY UNIVERSITY OF OREGON
UNIVERSITY OF SOUTHERN CALIFORNIA STANFORD UNIVERSITY UNIVERSITY OF HAWAII UNIVERSITY OF TOKYO UNIVERSITY OF UTAH WASHINGTON STATE UNIVERSITY UNIVERSITY OF WASHINGTON 


\section{Pacific Journal of Mathematics \\ Vol. 77, No. $1 \quad$ January, 1978}

Dan Amir, Chebyshev centers and uniform convexity ............... 1

Lawrence Wasson Baggett, Representations of the Mautner group. I ..... 7

George Benke, Trigonometric approximation theory in compact totally

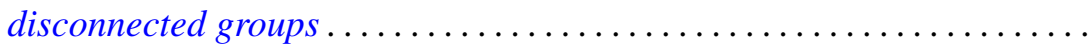

M. Bianchini, O. W. Paques and M. C. Zaine, On the strong compact-ported topology for spaces of holomorphic mappings ..................

Marilyn Breen, Sets with $(d-2)$-dimensional kernels

J. L. Brenner and Allen Kenneth Charnow, Free semigroups of $2 \times 2$

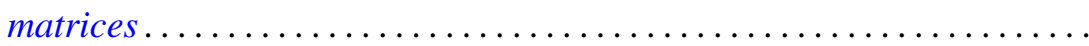

David Bressoud, A new family of partition identities .................

David Fleming Dawson, Summability of matrix transforms of stretchings

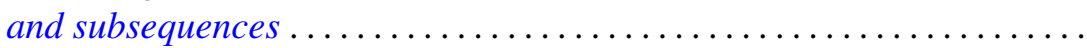

Harold George Diamond and Paul Erdôs, A measure of the nonmonotonicity

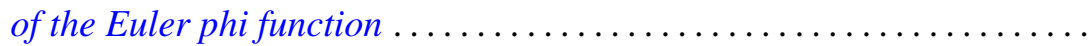

Gary Doyle Faulkner and Ronald Wesley Shonkwiler, Kernel dilation in reproducing kernel Hilbert space and its application to moment

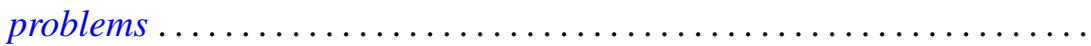

Jan Maksymilian Gronski, Classification of closed sets of attainability in the

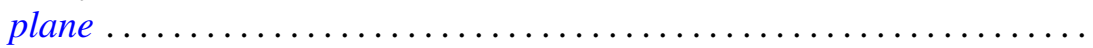

H. B. Hamilton and T. E. Nordahl, Semigroups whose lattice of congruences

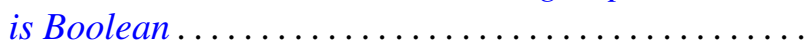

Harvey Bayard Keynes and D. Newton, Minimal $(G, \tau)$-extensions ...

Anthony To-Ming Lau, The Fourier-Stieltjes algebra of a topological

semigroup with involution.

B. C. Oltikar and Luis Ribes, On prosupersolvable groups ...

Brian Lee Peterson, Extensions of pro-affine algebraic groups ...

Thomas M. Phillips, Primitive extensions of Aronszajn spaces ...

Mehdi Radjabalipour, Equivalence of decomposable and 2-decomposable operators. .

M. Satyanarayana, Naturally totally ordered semigroups .

Fred Rex Sinal, A homeomorphism classification of wildly imbedded two-spheres in $S^{3}$

Hugh C. Williams, Some properties of a special set of recurring

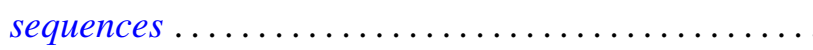

